\title{
Determinação de cumarina em extrato fluido e tintura de guaco por espectrofotometria derivada de primeira ordem
}

\author{
Adriana de Carvalho Osório e Jorge Luiz Seferin Martins* \\ Laboratório de Controle de Qualidade Físico-químico de Medicamentos e Cosméticos, Departamento de Farmácia, \\ Faculdade de Ciências Farmacêuticas, Universidade de São Paulo
}

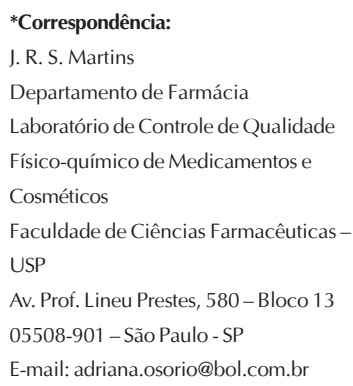

\section{INTRODUÇÃO}

O guaco (Mikania glomerata Sprengel) é uma planta medicinal brasileira empregada em medicamentos para tosse e problemas respiratórios (Panizza, 1997). Encontra-se comercializado, principalmente nas formas farmacêuticas de extrato fluido, tintura e xarope. Esta planta tem uso muito difundido, sendo usada pelos índios para picada de cobra e, até hoje, é amplamente utilizada na medicina popular como remédio para aliviar tosse, bronquite e resfriado (Di Stasi et al., 1994; Panizza, 1997).

Das atividades farmacológicas atribuídas à Mikania glomerata Sprengel, Oliveira et al. (1985) constataram a ação antiinflamatória do extrato fluido através do teste da atividade antiedema em pata do rato, induzida por carragenina e quantificada por pletismografia. Os resultados obtidos apresentaram aumento do volume percentual da pata de $75,4 \%$, após 300 minutos e de $81,6 \%$, comparando com o grupo controle. Essas inibições foram ligeiramente menores do que a produzida por fenilbutazona.

Leite et al. (1993) estudaram de modo comparativo os efeitos do extrato hidroalcoólico da Mikania glomerata Sprengel em relação aos produzidos pela cumarina (1,2benzopirano) encontrada na planta, submetendo a ensaios in vivo (edema de pata induzido pela carragenina em rato) e in vitro (preparações de jejuno de rato, íleo de cobaia e traquéia de rato utilizando acetilcolina e histamina como agonistas). Os resultados mostraram efeitos espasmolítico, antiinflamatório e broncodilatador do extrato e, também, da solução de cumarina. A diferença na intensidade dos efeitos farmacológicos observados indica que a cumarina contribuiu para o efeito farmacológico juntamente com outras substâncias químicas presentes no extrato.

Ruppelt et al. (1991) estudaram a atividade analgésica e antiinflamatória do chá de guaco, usando os respectivos ensaios de número de contorções em camundongos e difusão do corante azul de Evans no peritônio, segundo a técnica de Whittle modificada por Fernandes. O grupo de camundongos que ingeriu o chá de guaco apresentou inibição de contorções de $63,1 \%$ e difusão do corante de $48,92 \%$, em relação ao grupo controle. Esses resultados indicam que o guaco demonstra atividade analgésica e em menor intensidade atividade antiinflamatória.

Pereira et al. (1994) estudaram a atividade antiofídica da cumarina extraída da Mikania glomerata Sprengel frente ao veneno da jararaca (Bothrops jararaca). A sobrevivência dos animais foi avaliada em 6, 24 e 48 horas e registrada em porcentagem de animais vivos. A cumarina apresentou taxas de sobrevivência de $80 \%, 50 \%$ e $40 \%$, respectivamente aos intervalos de 6, 24 e 48 horas, enquanto, o grupo controle apresentou para os mesmos intervalos taxas de $30 \%, 0 \%$ e $0 \%$ de sobrivivência, respectivamente. 
Fierro et al. (1999) avaliaram frações obtidas do extrato etanólico de Mikania glomerata Sprengel para as propriedades antialérgica e antiinflamatória, no qual se obtiveram resultados que sugeriram que as frações estudadas são efetivas na inibição da inflamação imunológicas.

O estudo químico do guaco mostra as seguintes substâncias isoladas: cumarina (1,2-benzopirano), ácido caurenóico, ácido cinamolglandiflóroco, estigmasterol, friedelina, e lupeol (Oliveira et al., 1984; Vilegas et al., 1995; Veneziani et al., 1999).

A cumarina (1,2-benzopirano) foi escolhida como substância marcadora por ser um dos constituintes majoritários do vegetal e contribuir para o efeito farmacológico (Leite et al., 1993).

O objetivo deste trabalho foi desenvolver um método de doseamento de cumarina (1,2-benzopirano) por espectrofotometria derivada em extrato fluido e tintura de guaco, contribuindo para o controle de qualidade desta droga.

\section{MATERIAL E MÉTODOS}

\section{Material botânico}

As partes aéreas do vegetal foram fornecidas pelo Centro Pluridisciplinar de Pesquisas Químicas, Biológicas e Agrícolas da Unicamp. A planta foi identificada segundo a descrição da Farmacopéia Brasileira 1 ${ }^{\mathrm{a}}$ ed. (Silva, 1929) e pulverizada.

\section{Preparação de extratos}

Extrato fluido e tintura foram preparados de acordo com a Farmacopéia Brasileira $1^{\mathrm{a}}$ ed. (Silva, 1929). O líquido extrator utilizado foi álcool/água (2:1) (Lucas, 1942).

\section{Solução do padrão de cumarina}

Foi dissolvido 0,1 g de cumarina (1,2-benzopirano) em $5 \mathrm{~mL}$ de etanol em balão volumétrico de $50 \mathrm{~mL}$ e completou-se o volume com água destilada $(1 \mathrm{~mL}=2 \mathrm{mg}$ de cumarina).

\section{Construção da curva de calibração do padrão de cumarina}

Foram transferidos alíquotas da solução padrão de cumarina de 1,0 mL, 1,5 mL, 2,0 mL, 2,5 mL, 3, $0 \mathrm{~mL}, 3,5$ $\mathrm{mL}, 4,0 \mathrm{~mL}, 4,5 \mathrm{~mL}, 5,0 \mathrm{~mL}, 5,5 \mathrm{~mL}$ e $6,0 \mathrm{~mL}$ para balões volumétricos de $100 \mathrm{~mL}$. A cada balão adicionaram-se, aproximadamente, $20 \mathrm{~mL}$ de água destilada e $5 \mathrm{~mL}$ de acetato de chumbo $5 \%$. Completou-se o volume com água destilada. Filtrou-se, desprezando os primeiros $10 \mathrm{~mL}$, transferiu-se uma alíquota de $10,0 \mathrm{~mL}$ do filtrado para balão volumétrico de $50 \mathrm{~mL}$ e completou-se o volume com solução de $\mathrm{HCl}$ 0,1 M. A leitura da absorbância na derivada de primeira ordem de cada solução foi efetuada a $320 \mathrm{~nm}$, em Espectrofotômetro Shimadzu 1601 UV-Visível, pela técnica de zero-crossing. Empregou-se como branco a solução de $\mathrm{HCl} 0, \mathrm{M}$.

\section{Aplicação do método para extrato fluido e tintura de guaco}

Alíquotas de 2,0 mL de extrato fluido e 5,0 mL de tintura foram transferidas, respectivamente, para balões volumétricos de $100 \mathrm{~mL}$ e prosseguiu-se de acordo com o procedimento utilizado na curva de calibração do padrão. As leituras das absorbâncias na derivada de primeira ordem foram efetuadas a $320 \mathrm{~nm}$, comparando-se com solução padrão de cumarina a $12 \mu \mathrm{g} / \mathrm{mL}$. Foram feitas 10 determinações de cada uma das amostras para avaliação do desvio padrão relativo. Para extrato fluido foram repetidas 5 determinações após 2 semanas e mais 5 determinações após 4 semanas.

\section{Teste de recuperação}

Adicionou-se a determinada quantidade de extrato fluido quantidade de solução padrão de cumarina ( $2 \mathrm{mg} /$ $\mathrm{mL}$ ) na seguinte proporção: $\mathrm{I}-1,0 \mathrm{~mL}$ da solução padrão de cumarina $+2,0 \mathrm{~mL}$ do extrato fluido, II- $2,0 \mathrm{~mL}$ de solução padrão de cumarina $+2,0 \mathrm{~mL}$ de extrato fluido. $\mathrm{O}$ teste foi feito em duplicata, de acordo com o procedimento utilizado na preparação da curva de calibração do padrão. O cálculo para obtenção da porcentagem de recuperação foi determinado utilizando-se a concentração de alíquotas de $1,0 \mathrm{~mL}$ e 2,0 mL de solução padrão de cumarina e com alíquota de $2,0 \mathrm{~mL}$ de extrato fluido.

\section{Teste de linearidade}

O teste de linearidade foi feito preparando-se uma solução de cumarina a $1 \mathrm{mg} / \mathrm{mL}$ e adicionando-se alíquotas de $1,0 \mathrm{~mL}, 2,0 \mathrm{~mL}, 3,0 \mathrm{~mL}, 4,0 \mathrm{~mL}$ e $5,0 \mathrm{~mL}$ a $1,0 \mathrm{~mL}$ de extrato fluido e prosseguindo-se de acordo com o procedimento utilizado na preparação da curva de calibração do padrão.

\section{Estudo de interferências dos componentes do extrato fluido de guaco}

O estudo de interferência foi efetuado empregandose cromatografia preparativa. A fase móvel utilizada foi 
heptano/acetona (10:3) em fase estacionária sílica-gel 60. Prossegui-se, adicionando-se $1 \mathrm{~mL}$ de acetato de chumbo a $2 \mathrm{~mL}$ de extrato fluido, filtrou-se e o filtrado foi aplicado em linha na placa cromatográfica preparativa paralelamente ao padrão de cumarina. Foram reveladas duas porções da placa, com os respectivos reveladores

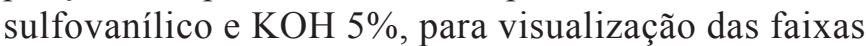
obtidas. As dez faixas resultantes foram separadas e diluídas em $10 \mathrm{~mL}$ de álcool/água 2:1 (veículo utilizado na preparação do extrato fluido). Efetuaram-se varreduras espectrais individuais de cada faixa resultante e sobrepostas na primeira ordem, juntamente com a varredura espectral do padrão de cumarina.

\section{RESULTADOS E DISCUSSÃO}

Os dados referentes à curva de calibração da solução do padrão de cumarina encontram-se à Figura 1, Tabela I. O coeficiente de linearidade da curva de calibração foi de 0,9951 indicando que o método é linear quando aplicado à substância pura (Brittain, 1998).

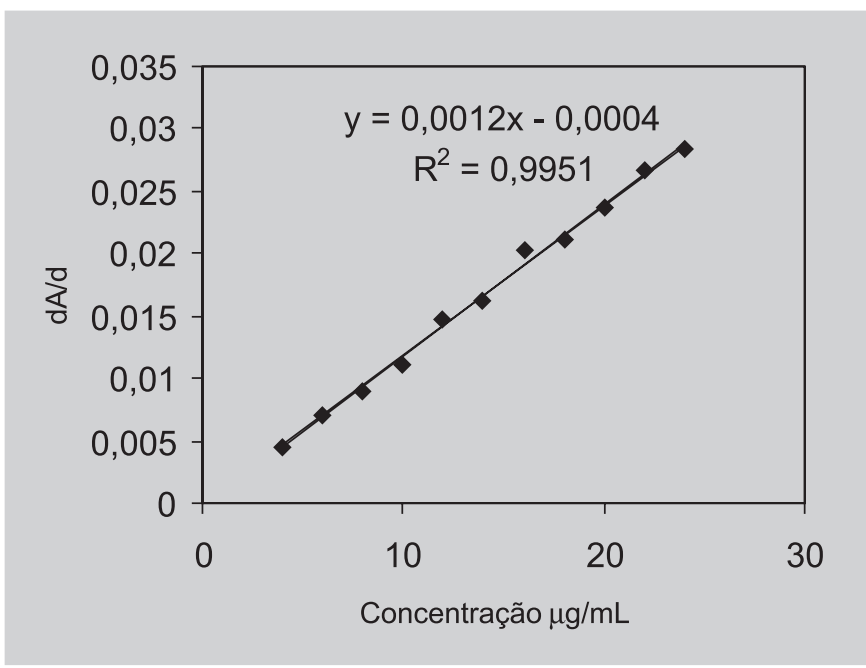

FIGURA 1 - Curva de calibração da solução padrão da cumarina, em $320 \mathrm{~nm}$, onde se obteve $\mathrm{y}=0,0012 \mathrm{x}-0,0004$, $\mathrm{R}^{2}=0,9951$ e $\mathrm{R}=0,9975$. Os valores obtidos são referentes à Tabela I.

O método desenvolvido por espectrofotometria derivada foi padronizado em bases experimentais. A adição de acetato de chumbo $5 \%$ e posterior filtração é necessária para precipitação de taninos. O pico máximo no espectro de primeira ordem para o extrato fluido e o padrão de cumarina foi de $290 \mathrm{~nm}$, mas a leitura foi estabelecida em $320 \mathrm{~nm}$ (Figura 2). Este comprimento de onda foi definido pelo estudo de interferência dos componentes do extrato fluido de guaco. A faixa que na placa cromatográfica se
TABELA I - Valores utilizados para a construção da curva da solução do padrão de cumarina pelo método da espectrofotometria derivada de primeira ordem

\begin{tabular}{lcc}
\hline & $\begin{array}{c}\text { Concentração do } \\
\text { padrão de cumarina } \\
\text { em } \boldsymbol{\mu g} / \mathbf{m L}\end{array}$ & $\begin{array}{c}\mathbf{d A} / \mathbf{d} \boldsymbol{\lambda} \text { na } \\
\text { absorbância } \\
\text { em 320nm }\end{array}$ \\
\hline 1 & 4 & $-0,0045$ \\
2 & 6 & $-0,0071$ \\
3 & 8 & $-0,0090$ \\
4 & 10 & $-0,0111$ \\
5 & 12 & $-0,0148$ \\
6 & 14 & $-0,0162$ \\
7 & 16 & $-0,0203$ \\
8 & 18 & $-0,0212$ \\
9 & 20 & $-0,0236$ \\
10 & 22 & $-0,0266$ \\
11 & 24 & $-0,0283$ \\
\hline
\end{tabular}

Obs: A curva de calibração foi construída em valores absolutos de $\mathrm{dA} / \mathrm{d} \lambda$

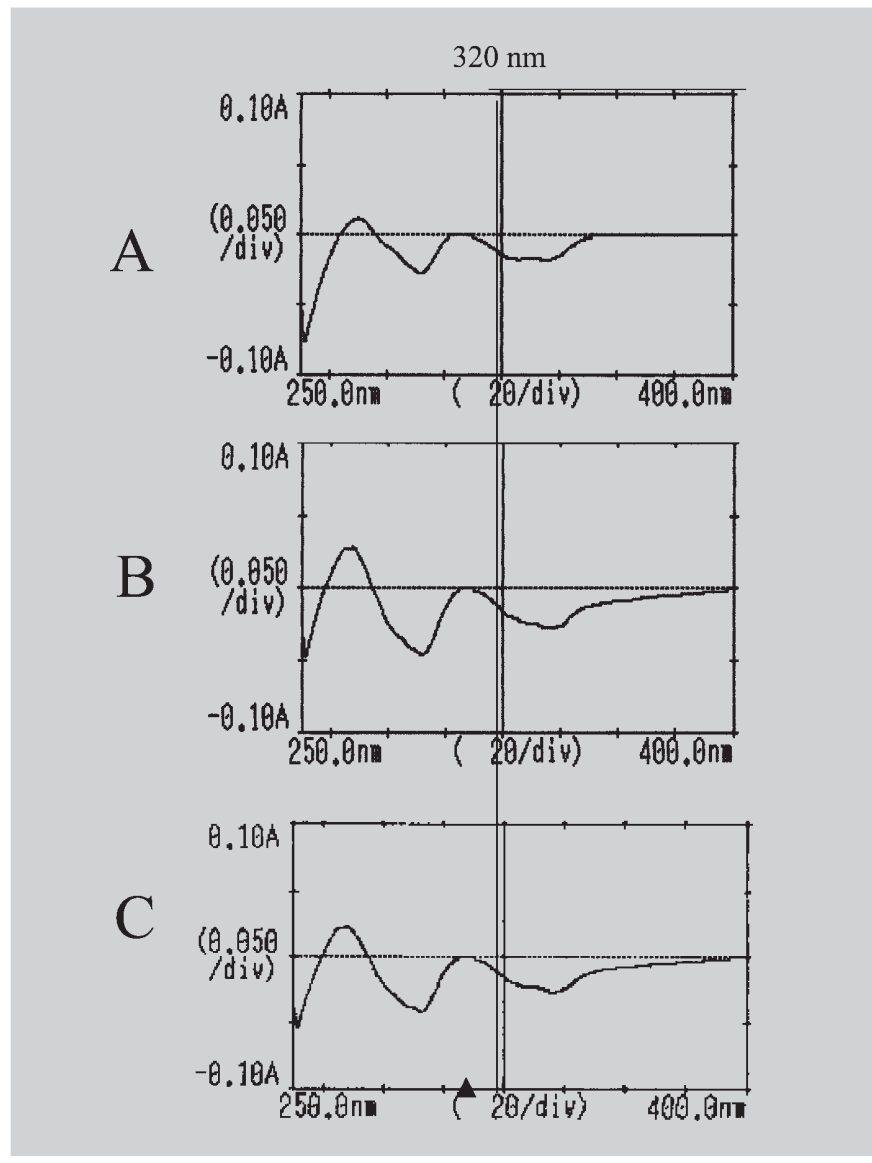

FIGURA 2 - Derivadas de primeira ordem das varreduras espectrais: A - solução padrão de cumarina, B - extrato fluido de guaco, $\mathrm{C}$ - tintura de guaco. 
TABELA II - Resultados estatísticos do doseamento por espectrofotometria derivada de primeira ordem a $320 \mathrm{~nm}$ para extrato fluido e tintura de guaco

\begin{tabular}{llccc}
\hline & Extrato fluido & $\begin{array}{c}\text { Extrato fluido após } \\
2 \text { semanas }\end{array}$ & $\begin{array}{c}\text { Extrato fluido após } \\
4 \text { semanas }\end{array}$ & Tintura \\
\hline Média $(\mathrm{mg} / \mathrm{mL})^{*}$ & $3,79^{* *}$ & $3,82^{* * *}$ & $3,84 * * *$ & $1,10^{* *}$ \\
Desvio padrão & 0,08 & 0,10 & 0,053 & 0,019 \\
Desvio padrão relativo & 0,021 & 0,026 & 0,013 & 0,017 \\
Coef. de variação & $2,10 \%$ & $2,60 \%$ & $1,38 \%$ & $1,72 \%$ \\
\hline
\end{tabular}

Obs:mg de cumarina por $\mathrm{mL}$ de extrato, ${ }^{*} * \mathrm{n}=10, * * * \mathrm{n}=5$

identificava com padrão (Figura 3-D) teve coincidência com varredura espectral da solução do padrão de cumarina (Figura 2-A). O pico apresentado pela cumarina localizase em $290 \mathrm{~nm}$, mas neste comprimento de onda há outra faixa que apresenta absorção (Figura 3). Em $320 \mathrm{~nm}$ apresentou leitura somente para faixa que corresponde ao padrão e neste ponto os espectros das demais faixas se anulam, portanto a técnica escolhida para determinar a concentração foi zero-crossing, no qual o valor absoluto da absorbância é medido a partir da linha da abscissa do eixo de anulação e onde as outras substâncias se anulam.

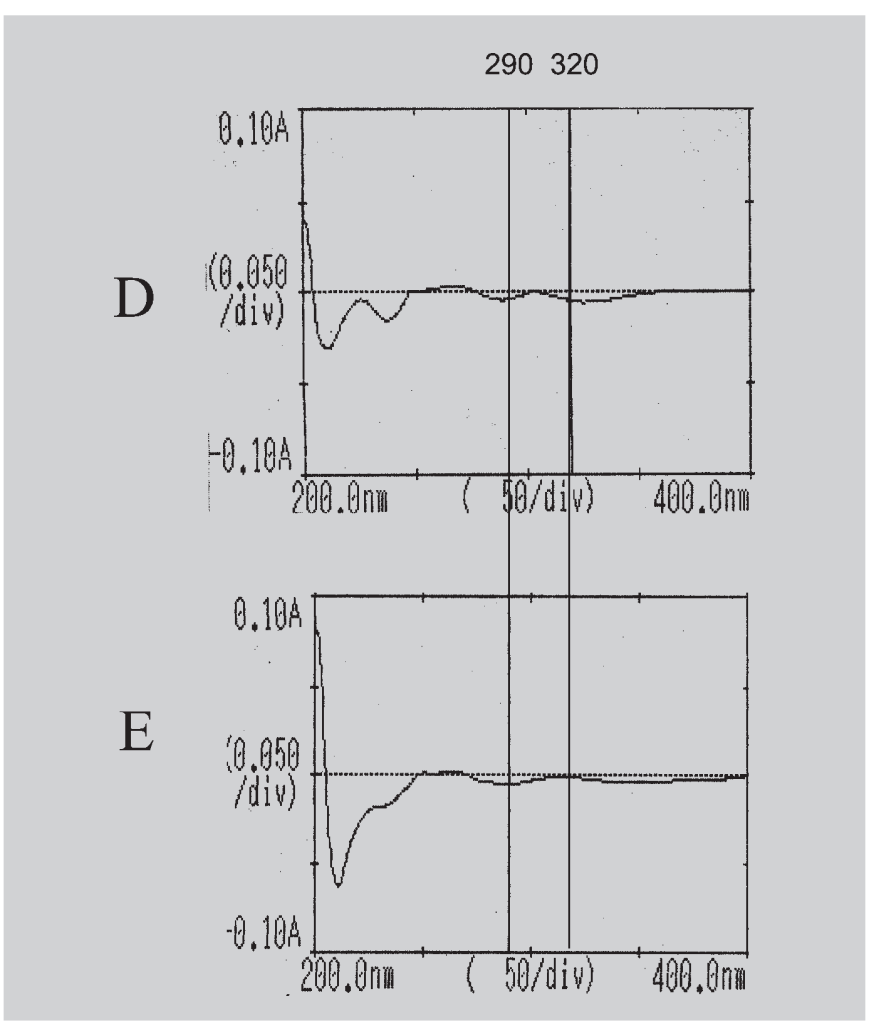

FIGURA 3 - Derivadas de primeira ordem das varreduras espectrais das soluções obtidas das faixas resultantes da cromatografia preparativa em camada delgada. D - faixa correspondente ao padrão de cumarina; $\mathrm{E}$ - faixa que apresentou leitura em $290 \mathrm{~nm}$.
Os resultados estatísticos obtidos para o método da espectrofotometria derivada (Tabela II) indicam: precisão por apresentar desvio padrão relativo menor que 1; exatidão por apresentar teste de recuperação em torno de $102 \%$ (Tabela III) e reprodutibilidade pelo valor de desvio padrão obtido (Brittain, 1998).

TABELA III -Valores obtidos no teste de recuperação do extrato fluido de guaco pelo método de espectrofotometria derivada de primeira ordem.

\begin{tabular}{lc}
\hline & \% recuperação \\
\hline I & 103,37 \\
II & 102,50 \\
\hline
\end{tabular}

Obs: Os valores são médias de duas determinações, com diferença $<1 \%$.

Para o estudo de linearidade, testou-se a correlação entre quantidade conhecida de padrão adicionado ao extrato fluido e a quantidade encontrada em análise. O coeficiente de correlação da reta encontrado foi de 0,9987 indicando que o método é linear para aplicação no extrato fluido (Figura IV, Tabela IV).

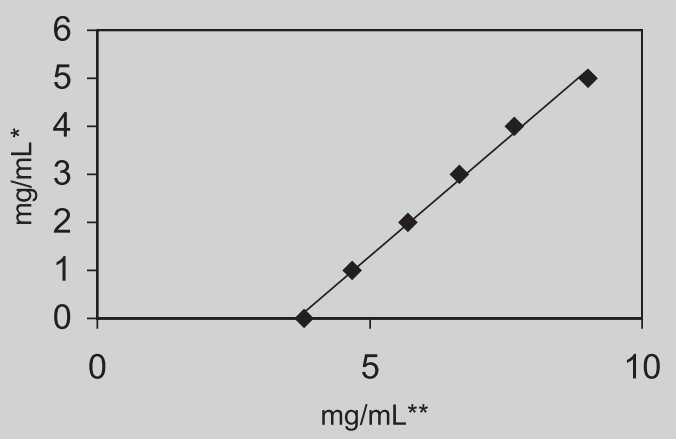

FIGURA 4 - Curva do teste de linearidade em que obtevese $\mathrm{R}^{2}=0,9976$ e $\mathrm{R}=0,9987$. (*) e (**) correspondem, respectivamente, aos valores em $\mathrm{mg} / \mathrm{mL}$ do padrão de cumarina adicionada ao extrato e $\mathrm{mg} / \mathrm{mL}$ determinado na análise. 
TABELA IV - Concentrações calculadas no teste de linearidade empregando método da espectrofotometria derivada no extrato fluido de guaco

\begin{tabular}{cc}
\hline $\begin{array}{c}\text { Quantidade de cumarina } \\
\text { adicionada ao extrato } \\
\text { fluido de guaco } \\
\text { em } \mathbf{~ m g / m l}\end{array}$ & $\begin{array}{c}\text { Concentração } \\
\text { determinada } \\
\text { no extrato fluido } \\
\text { de guaco em } \mathbf{~ m g / m L}\end{array}$ \\
\hline 0 & 3,80 \\
1 & 4,67 \\
2 & 5,70 \\
3 & 6,64 \\
4 & 7,66 \\
5 & 9,01 \\
\hline
\end{tabular}

Obs: Média de duas determinações, com diferença $<1 \%$.

\section{CONCLUSÃO}

Os métodos usuais descritos na literatura para determinação de especificidade e linearidade utilizados para substâncias incorporadas na forma farmacêutica são de difícil aplicação em extratos vegetais, uma vez que recorrem à preparação de um placebo e incorporação do princípio ativo em concentração determinada; isto é impossível para extratos vegetais, porque nas plantas há mistura natural de compostos.

A espectrofotometria por derivada de primeira ordem, pelo coeficiente de linearidade alto $(0,9987)$, quando de sua aplicação em extrato fluido de guaco, mostrou-se útil para a aplicação em extratos vegetais.

\section{AGRADECIMENTOS}

Os autores agradecem à Fundação de Amparo à Pesquisa do Estado de São Paulo (FAPESP), pelo auxilio financeiro, e ao Centro Pluridisciplinar de Pesquisas Químicas, Biológicas e Agrícolas (CPQBA), da Unicamp, pelo fornecimento do material vegetal.

\section{ABSTRACT \\ Determination of coumarin in fluid extract and tinture of "guaco" by first derivative spectrophotometry}

The objective of this work was to develop a method for coumarin (1,2-benzopyran) dosage in fluid extract and tinture of "guaco" (Mikania glomerata Sprengel). First derivative spectrophotometry was developed and proved to be accurate, exact, reprodutive and of easy execution.

UNITERMS: Mikania glomerata. Guaco. Coumarin. First Derivative spectrophotometry. Fluid extract.

\section{REFERÊNCIAS BIBLIOGRÁFICAS}

BRITTAIN, H. Validação de métodos analíticos não cromatográficos. Pharm. Technol., Ed. Bras., São Paulo, v.2, p.4-9, 1998.

DI STASI, L. C. et al. Plantas medicinais da medicina popular no município de Botucatu-SP. In: SIMPÓSIO DE PLANTAS MEDICINAIS DO BRASIL, 13., Fortaleza, 1994. Livro de Resumos. Fortaleza: CNPq, 1994. Res. 320.

FIERRO, I. M.; SILVA, A. C. B.; LOPES, C. S.; MOURA, R. S.; FIDALGO, C. B. Studies on the anti-allergic activitity of Mikania glomerata. J. Ethnopharmacol., Shannon, v.66, n.1, p.19-24, 1999.

LEITE, M. G. R.; SOUZA, C. L.; SILVA, M. A. M.; MOREIRA, L. K. A.; MATOS, F. J. A.; VIANA, G. S. B. Estudo farmacológico comparativo de Mikania glomerata Sprengel (guaco), Justicia pectoralis Jacq (anador) e Torresea cearensis (cumaru). Rev. Bras. Farm., Rio de Janeiro, v.74, n.1, p.12-15, 1993.

LUCAS, V. Estudo farmacognóstico do guaco. Rev. Flora Med., Rio de Janeiro, v.9, n.3, p.101-132, 1942.

OLIVEIRA, F.; ALVARENGA, M. A.; AKISUE, G.; AKISUE, M. K. Isolamento e identificação de componentes químicos de Mikania glomerata Sprengel e de Mikania laevigata Schutz Bip. ex Baker. Rev. Farm. Bioquim. Univ. São Paulo, São Paulo, v.20, n.2, p.169-183, 1984.

OLIVEIRA, F.; OGA, S.; AKISUE, G.; AKISUE, M. K. Parâmetros físicos e químicos e efeito antiedema dos extratos fluidos de guaco (Mikania glomerata Sprengel) e de guaco de mato (Mikania laevigata Schutz Bip. ex Baker). An. Farm. Quim., São Paulo, v.25, n.1/2, p.5054, 1985.

PANIZZA, S. Plantas que curam: cheiro de mato. São Paulo: IBRASA, 1997. p.117-118. 
PEREIRA, N. A.; PEREIRA, B. M. R.; NASCIMENTO, M. C.; PARENTE, J. P.; MORS, W. B. Pharmacological screening of plants recommended by folk medicine as anti-snake venom. IV. Proction against jararaca venom by isolated constituints. Planta Med., Stuttgart, v.60, p.99100, 1994.

RUPPELT, B. M.; PEREIRA, E. F. R.; GONÇAVES, L .G.; PEREIRA, A. N. Pharmacological screening of plants recommended by folk medicine as anti-snake venom. I. Analgesic and anti-inflammatory activities. Mem. Inst. Oswaldo Cruz, Rio de Janeiro, v.86, n.2, p.203-205, 1991.

SILVA, R. A. Pharmacopeia dos Estados Unidos do Brasil. São Paulo: Ed. Nacional, 1929. p.384-387, 425, 495, 893-894, 929.
VENEZIANI, R. C. S.; CAMILO, D.; OLIVEIRA, R. Constituents of Mikania glomerata Sprengel. Biochem. Syst. Ecol., Oxford, v.27, n.1, p.99-102, 1999.

VILEGAS, J. H. Y.; MARCHI, E.; LANÇAS, F. M. Extration of low-polarity compounds (with amphasis on coumarin and Kaurenoic acid) from Mikania glomerata ('guaco') leaves. Phytochem. Anal., Chischester, v.8, p.266-270, 1997.

Recebido para publicação em 15 de julho de 2003 Aceito para publicação em 08 de outubro de 2004. 